\title{
CThCC4.pdf
}

\section{Local tuning of photonic crystal cavities using chalcogenide glasses}

\author{
Andrei Faraon *, Dirk Englund \\ Department of Applied Physics, Stanford University, Stanford, CA, 94305, USA \\ Douglas Bulla, Barry Luther-Davies \\ Centre for Ultrahigh-bandwidth Devices for Optical Systems (CUDOS), \\ Laser Physics Centre, Australian National University, Canberra, ACT, 0200, Australia \\ Benjamin J. Eggleton \\ Centre for Ultrahigh-bandwidth Devices for Optical Systems (CUDOS), \\ School of Physics, University of Sydney, Sydney, NSW, 2006, Australia \\ Nick Stoltz, Pierre Petroff \\ Department of Electrical and Computer Engineering, \\ University of California, Santa Barbara, CA 93106 \\ Jelena Vučković \\ E. L. Ginzton Laboratory, Stanford University, Stanford, CA, 94305, USA
}

\begin{abstract}
We developed a method to locally tune refractive index in photonic crystals. The technique, based on photodarkening of chalcogenide glasses, enables $3 \mathrm{~nm}$ resonance tuning of GaAs photonic crystal cavities operating at $940 \mathrm{~nm}$.
\end{abstract}

Photonic crystals represent one of the most promising platforms for on chip integration [1] of optical components. However, they are very sensitive to fabrication imperfection, so a practical method to post-tune their optical properties is needed. Here we present such a method based on chalcogenide glasses. Chalcogenide glasses quasi-permanently change their optical properties when illuminated with light above their band gap, and have been used to tune optical devices as quantum cascade lasers [2]. The tuning of PCs devices directly fabricated in chalcogenide glasses has already been shown in Ref.[3], but many other applications rely on PC fabricated in other materials such as group IV and III-V semiconductors.

In our approach, a photosensitive chalcogenide glass layer is deposited on prefabricated GaAs/InAs devices. Linear three-hole defect [4] PC cavities were first fabricated in a $150 \mathrm{~nm}$ thick GaAs membrane containing a central layer of InAs quantum dots (QDs) as described in Ref.[5]. Arsenic trisulphide films with thickness between $30 \mathrm{~nm}$ and $100 \mathrm{~nm}$ were deposited onto the photonic crystals using thermal evaporation.

The experiment was performed at cryogenic temperature (less than $\sim 60 K$ ) to obtain luminescence from the embedded InAs quantum dots, as needed for quantum information processing applications. This illustrates that the method works at low temperatures, though we stress that it is applicable to room temperature nanophotonic circuits. The sample was placed inside a continuous-flow liquid helium cryostat at $10 \mathrm{~K}$ and the $\mathrm{QD}$ photolumi-

*Electronic address: faraon@stanford.edu nescence was used to measure the cavity resonance. A confocal microscope setup and a laser tuned at $780 \mathrm{~nm}$ excited quantum dot luminescence while a spectrometer monitored the signal. A $543 \mathrm{~nm}$ HeNe laser $(1 \mu W)$ focused to $\sim 1 \mu \mathrm{m}^{2}$ through the same confocal setup was used for photodarkening of the $\mathrm{As}_{2} \mathrm{~S}_{3}$ layer (Fig.1). This wavelength was chosen because it is close to the $527 \mathrm{~nm}$ bandgap of $\mathrm{As}_{2} \mathrm{~S}_{3}$.

The thickness of the $\mathrm{As}_{2} \mathrm{~S}_{3}$ influences both the quality factor of the cavity and the maximum tuning range. For this reason we experimented with three different thicknesses: 30, 60 and $100 \mathrm{~nm}$ (samples S30, S60 and S100). For each sample, the spectrum of the cavities was recorded before and after the deposition of the chalcogenide layer. For samples S60(S30), the deposition caused the quality factor to degrade by $\sim 5 \%(30 \%)$ from an average value of $\sim 8500(10000)$ while the resonant wavelength shifted by $\sim 40 \mathrm{~nm}(28 \mathrm{~nm})$. For sample S100 the Q degradation was more severe, from $\sim 6500$ to $\sim 1000$ and for this reason we mainly concentrate on samples S30 and S60.

With the chips mounted in the cryostat, we focused the $543 \mathrm{~nm}$ laser on the PC cavities for a fixed time and recorded the cavity spectrum. For sample S60, the cavity resonance shifted by up to $3 \mathrm{~nm}$ as shown in Fig.2(a). For $1 \mu \mathrm{W}$ of green laser power focused on a spot size of $\sim 1 \mu \mathrm{m}^{2}$, the cavity tuning rate levels off after about 20 minutes, as shown in Fig.2(b). This saturation time is inversely proportional to the energy flux incident on the sample surface. During the tuning process the quality factor degraded by $20 \%$. The maximum tuning range dependends on the thickness of the chalcogenide layer. For $30 \mathrm{~nm}$ and $100 \mathrm{~nm}$, a tuning range of $1 \mathrm{~nm}$ (Fig.2(b)) and $4 \mathrm{~nm}$ was observed, respectively. The change of the cav- 


\section{CThCC4.pdf}

ity resonance was stable after the green laser was turned off.
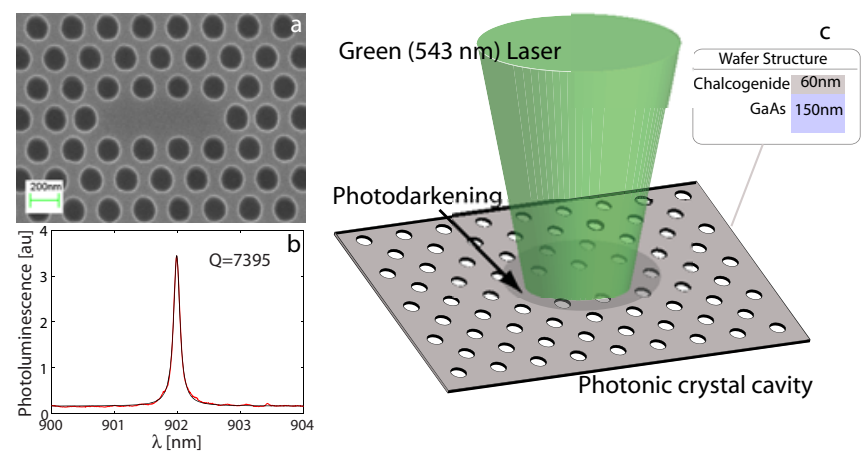

FIG. 1: (a) Scanning electron microscope image of the photonic crystal cavity fabricated in GaAs before the deposition of $\mathrm{As}_{2} \mathrm{~S}_{3}$. (b) Cavity spectrum before the chalcogenide deposition indicating a quality factor $\mathrm{Q}=7395$. (c)Schematic of the method for local cavity tuning. A layer of $\mathrm{As}_{2} \mathrm{~S}_{3}$ is deposited on top of the photonic crystal cavity. Then a laser tuned close to the $\mathrm{As}_{2} \mathrm{~S}_{3}$ band gap is focused on the cavity, increasing the effective refractive index and causing a resonance red-shift.
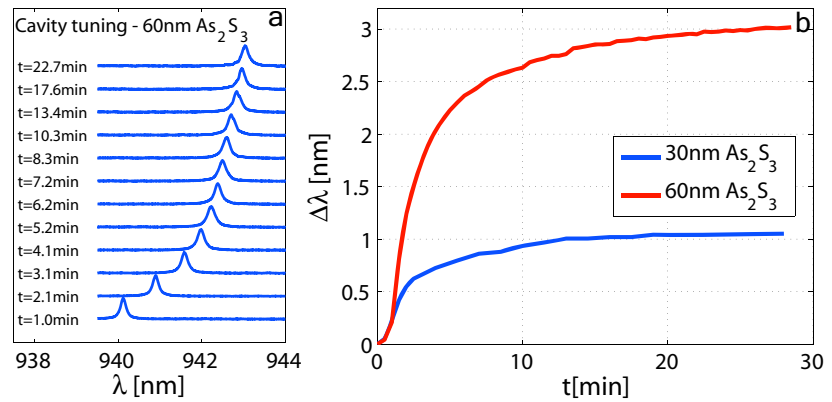

[1] Susumu Noda, Alongkarn Chutinan, and Masahiro Imada. Trapping and emission of photons by a single defect in a photonic bandgap structure. Nature, 407:608, 2000.

[2] Shanshan Song, Scott S. Howard, Zhijun Liu, Afusat O. Dirisu, Claire F. Gmachl, and Craig B. Arnold. Mode tuning of quantum cascade lasers through optical processing of chalcogenide glass claddings. Applied Physics Letters, 89(041115), 2006.

[3] Michael W. Lee, Christian Grillet, Cameron L.C. Smith, David J. Moss, Benjamin J. Eggleton, Darren Freeman, Barry Luther-Davies, Steve Madden, Andrei Rode, Yinlan Ruan, and Yong hee Lee. Photosensitive post tuning of
FIG. 2: (a)Spectra showing the shift of the cavity resonance because of the photodarkening of the $60 \mathrm{~nm}$ thick chalcogenide layer. (b) Time dependence of the cavity resonance for $60 \mathrm{~nm}$ and $30 \mathrm{~nm} \mathrm{As}_{2} \mathrm{~S}_{3}$ during the tuning process.

For our experiment, the smallest area that can be locally tuned is limited by the focus size of the laser beam $\left(\sim 1 \mu m^{2}\right)$. The locality of the technique allows for independent tuning of interconnected optical components on photonic crystal chips. The method is not only suitable for GaAs devices, but can possibly be implemented with any other materials, including silicon nanophotonic circuits. Also, the $\mathrm{As}_{2} \mathrm{~S}_{3}$ can easily be replaced by other types of chalcogenide glasses or other photosensitive materials depending on the specific application.

In conclusion, we have shown that $\mathrm{As}_{2} \mathrm{~S}_{3}$ can be combined with semiconductor photonic crystals to create nanophotonic devices whose optical properties can be independently fine-tuned on the same chip. This technique is relevant for fabrication of integrated nanophotonic circuits for classical and quantum information processing, including applications such as filtering, multiplexing, optical storage, fine-tuning of modulators and lasers, and local tuning of distinct PC cavities on GaAs/InAs chips for quantum optics.

Financial support was provided by ONR Young Investigator Award, the MURI Center for photonic quantum information systems (ARO/DTO program No. DAAD19-03-1-0199) and NSF Grant No. CCF-0507295. Work was performed in part at the Stanford Nanofabrication Facility of NNIN supported by the National Science Foundation under Grant ECS-9731293. CUDOS is an Australian Research Council Centre of Excellence. 